\title{
Nanogram Amounts of Salicylic Acid Produced by the Rhizobacterium Pseudomonas aeruginosa 7NSK2 Activate the Systemic Acquired Resistance Pathway in Bean
}

\author{
Geert De Meyer, ${ }^{1}$ Kristof Capieau, ${ }^{1}$ Kris Audenaert, ${ }^{1}$ Antony Buchala, ${ }^{2}$ Jean-Pierre Métraux, ${ }^{2}$ and \\ Monica Höfte ${ }^{1}$ \\ ${ }^{1}$ Lab of Phytopathology, Faculty of Agricultural and Applied Biological Sciences, University Gent, Coupure \\ links, 653, B-9000 Gent, Belgium; ${ }^{2}$ Institut de Biologie Végétale, Rue Albert-Gockel 3, Université \\ de Fribourg, $\mathrm{CH}-1700$ Fribourg, Switzerland \\ Accepted 3 February 1999.
}

\begin{abstract}
Root colonization by specific nonpathogenic bacteria can induce a systemic resistance in plants to pathogen infections. In bean, this kind of systemic resistance can be induced by the rhizobacterium Pseudomonas aeruginosa 7NSK2 and depends on the production of salicylic acid by this strain. In a model with plants grown in perlite we demonstrated that Pseudomonas aeruginosa 7NSK2induced resistance is equivalent to the inclusion of $1 \mathrm{nM}$ salicylic acid in the nutrient solution and used the latter treatment to analyze the molecular basis of this phenomenon. Hydroponic feeding of $1 \mathrm{nM}$ salicylic acid solutions induced phenylalanine ammonia-lyase activity in roots and increased free salicylic acid levels in leaves. Because pathogen-induced systemic acquired resistance involves similar changes it was concluded that 7NSK2-induced resistance is mediated by the systemic acquired resistance pathway. This conclusion was validated by analysis of phenylalanine ammonia-lyase activity in roots and of salicylic acid levels in leaves of soil-grown plants treated with Pseudomonas aeruginosa. The induction of systemic acquired resistance by nanogram amounts of salicylic acid is discussed with respect to long-distance signaling in systemic acquired resistance.
\end{abstract}

Additional keywords: Botrytis cinerea, induced systemic resistance.

Induced systemic resistance (ISR) refers to the state of enhanced resistance to pathogen infection that is activated by biotic or abiotic agents (Kloepper et al. 1992). In this perspective, ISR encompasses the strictly defined phenomenon of systemic acquired resistance (SAR) that occurs after a localized pathogen infection and is associated with distinct changes in the plant (Ryals et al. 1996). Development of SAR is mediated by a mobile signal that originates from the site of pathogen infection. For a long time the secondary plant metabolite salicylic acid (SA) was thought to fulfill this function. In some

Corresponding author: Monica Höfte; Telephone: +32 9264 6018; Fax: +329264 6238; E-mail: Monica.Hofte@ rug.ac.be plants, SA accumulates to several $\mu \mathrm{g} \mathrm{g}^{-1}$ leaf around localized pathogen infections and is subsequently detected in phloem and in distant plant parts, where it precedes the onset of SAR (Malamy et al. 1990; Métraux et al. 1990). Studies with radiolabeled SA or precursors of SA confirmed SA transport from the site of pathogen infection to distant plant parts but also demonstrated that the transported SA is only partially responsible for the increased SA levels in distant plant parts (Shulaev et al. 1995; Mölders et al. 1996). In consequence, synthesis of additional SA in distant plant parts seems essential for expression of SAR. This correlates with the fact that SAR cannot be expressed in transgenic plant parts that either degrade SA because they express the $n a h G$ gene that encodes a salicylate hydroxylase (NahG plants; Vernooij et al. 1994) or are impaired in SA synthesis through epigenetic suppression of phenylalanine ammonia-lyase (PAL; EC 4.3.1.5), a major enzyme in SA synthesis (Pallas et al. 1996). In spite of the clear association of systemic transport of SA and development of SAR, a role for SA as primary long-distance signal for SAR is still questioned. Firstly, because the mobile signal for SAR is generated in transgenic plant parts that do not allow substantial accumulation of SA (Vernooij et al. 1994; Pallas et al. 1996) and secondly because, in cucumber, the mobile signal for SAR is generated before significant accumulation of SA (Smith-Becker et al. 1998). These arguments and the observation that accumulation of SA in the phloem of cucumber is preceded by an increase in PAL activity (SmithBecker et al. 1998) suggest that a still unknown primary signal induces synthesis of SA that in turn functions as a secondary signal for SAR.

ISR also encompasses the increased resistance that is due to the colonization of plant roots by specific strains of nonpathogenic rhizobacteria (van Loon et al. 1998). Although ISR by rhizobacteria and SAR result in a similar increase of resistance it seems unlikely that nonpathogenic rhizobacteria activate the SAR signal transduction pathway because the latter is essentially activated after a pathogen infection. However, in iron-limited environments such as the rhizosphere most of the rhizobacteria that induce systemic resistance produce SA (Maurhofer et al. 1994; Buysens et al. 1996; Leeman et al. 
1996; Press et al. 1997), which could trigger the SAR signal transduction pathway. For some strains this possibility was rejected. ISR by Pseudomonas fluorescens WCS417 was still observed under conditions that excluded SA production by the rhizobacteria on the roots (Leeman et al. 1996) and SAdeficient mutants of Serratia marcescens 90-166 retained the same capacity to induce systemic resistance as the wild-type strain (Press et al. 1997). This demonstrates that SA production by these strains is not essential for ISR. The observation that $P$. fluorescens WCS417 and $S$. marcescens 90-166 induce systemic resistance in NahG plants (Pieterse et al. 1996; Press et al. 1997) confirms that ISR by these strains is independent of the SAR signal transduction pathway. However, the observation that SA-deficient mutants of $P$. aeruginosa 7 NSK2 lost the ability to induce resistance to Botrytis cinerea in bean (De Meyer and Höfte 1997) and to tobacco mosaic virus (TMV) in tobacco (G. De Meyer, K. Audenaert, and M. Höfte, unpublished) demonstrates that some rhizobacteria depend on SA to induce systemic resistance. This is supported by the conversion of $P$. fluorescens $\mathrm{P} 3$ to a systemic resistance-inducing strain by the introduction of SA biosynthetic genes (Maurhofer et al. 1998).

The objective of this study was to verify whether the SAR signal transduction pathway mediates the SA-dependent ISR by $P$. aeruginosa 7NSK2. We therefore estimated the amount of SA involved in ISR by 7NSK2 and applied this amount to roots of bean plants grown on perlite and nutrient solution to study subsequent biochemical changes related to SAR. Results obtained in this model were validated for soil-grown plants treated with $P$. aeruginosa.

\section{RESULTS}

Systemic resistance to $B$. cinerea induced by $P$. aeruginosa strains and SA.

Bean plants were grown on perlite and half-strength Hoagland solution and detached first leaves were challenged with $B$. cinerea to assay for induced resistance. All inoculations resulted in development of $B$. cinerea in the tissue under the inoculum drop but only part of the infections grew out of this zone and became a typical, gray, spreading $B$. cinerea lesion. The incidence of spreading lesions (about $50 \%$ on control leaves) was significantly reduced on leaves from plants whose roots were treated with $P$. aeruginosa 7 NSK2 (Fig. 1A). Given the spatial separation of 7NSK2 (roots) and $B$. cinerea (leaf) this effect is due to ISR. Root treatment with KMPCH, a pyoverdin- and pyochelin-deficient mutant of 7NSK2, also induced systemic resistance while treatment with MPFM1-569, deficient in production of pyoverdin, pyochelin, and SA, did not (Fig. 1A). This confirms the essential role of SA in ISR by 7NSK2 that was previously reported for soil-grown plants (De Meyer and Höfte 1997). A significant reduction in the incidence of spreading lesions was also observed for plants grown with nutrient solutions containing SA (Fig. 1B). In the $1 \mathrm{nM}$ to $100 \mu \mathrm{M}$ SA range, ISR was evident for all concentrations tested, except for 100 nM SA. All effective SA concentrations reduced the incidence of spreading lesions to the same extent as treatment of the roots with 7NSK2. This indicates that the SA-dependent ISR by $P$. aeruginosa 7NSK2 can be mimicked by application of SA to roots.
Amount of SA involved in ISR by $P$. aeruginosa 7NSK2.

The role of SA in ISR by 7NSK2 was further studied with the 7NSK2 mutant KMPCH, which produces SA and induces resistance (Fig. 1A) but does not produce pyochelin and pyoverdin, two compounds that are not essential for ISR by 7NSK2. Because the KMPCH root density in the perlite model (about $2 \times 10^{8} \mathrm{CFU} \operatorname{root}^{-1}$ ) was about 1,000 times higher than in vivo (De Meyer and Höfte 1997), we first investigated the effect of KMPCH root density on ISR by decreasing the KMPCH root density in our model. The KMPCH root density did not decrease below $2 \times 10^{8} \mathrm{CFU}$ root $^{-1}$ when the amount of bacteria applied to the root was decreased. Even an application of approximately $10^{3} \mathrm{CFU}$ per root resulted in a density of $2 \times 10^{8} \mathrm{CFU}$ plant $^{-1}$ root $48 \mathrm{~h}$ after inoculation. Most likely the sterility of the roots at the time of inoculation allows KMPCH to occupy all available niches until the maximal level of colonization is reached. In vivo, competition from other microorganisms prevents such complete root colonization by KMPCH. Therefore, we reduced KMPCH root density proportionally by inoculating roots with a mix of $\mathrm{KMPCH}$ and a diluting strain. Of course, the diluting strain should not affect ISR by KMPCH and should thus not produce SA, induce systemic resistance, or affect SA produced by KMPCH. MPFM1-569 (Fig. 1A) does not fulfill the last requirement because it is able to convert SA to pyochelin.

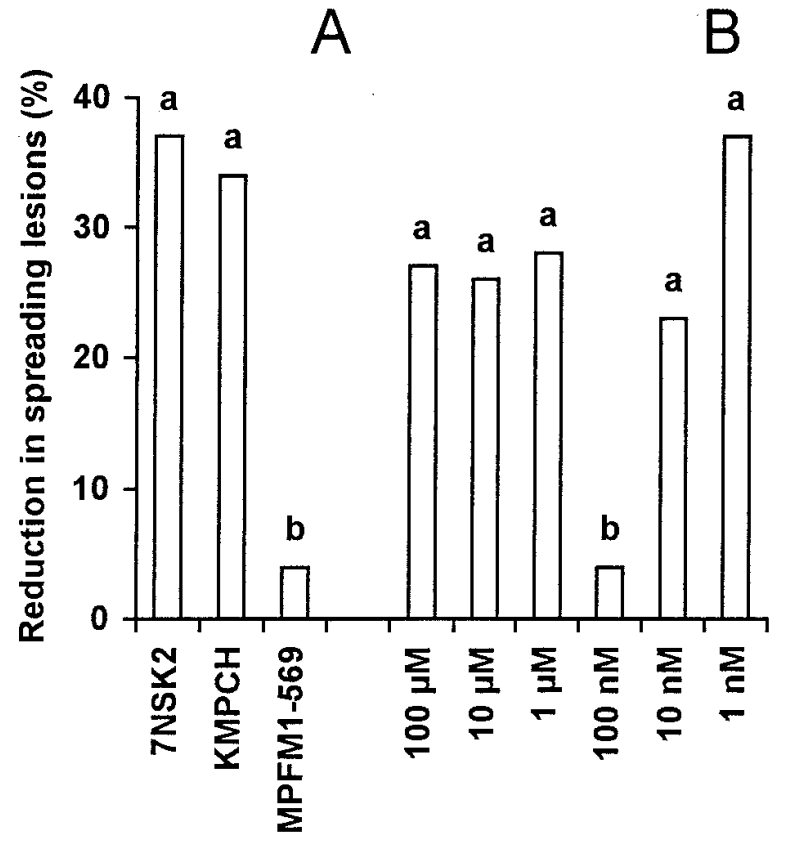

Treatment $[S A]$ in nutrient solution

Fig. 1. Effect of root treatment with (A) Pseudomonas aeruginosa strains or (B) inclusion of salicylic acid (SA) in nutrient solution on the number of spreading Botrytis cinerea lesions on the first leaves of bean plants grown on perlite and a half-strength Hoagland solution. Leaves were inoculated with $1010-\mu \mathrm{l}$ drops of a $10^{6} \mathrm{~B}$. cinerea conidia $\mathrm{ml}^{-1}$

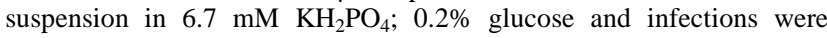
scored after 4 to 5 days. Results are presented as a reduction in spreading lesions relative to the number of spreading lesions in control plants. Data are means of (A) two or (B) four experiments each with 10 leaves per treatment. Bars with different letters are statistically different by logistic regression analysis at $P=0.05$. Bars marked with 'b' are not different from the control. 
Therefore, a new mutant of KMPCH was constructed by gene replacement of the KMPCH analog of the pchA gene, essential for the synthesis of SA (Serino et al. 1995), by a mutated copy. The resulting strain, KMPCH-567, like its parental strain deficient in the conversion of SA to pyochelin, did not produce SA (results not shown) and consequently did not induce systemic resistance (Fig. 2). KMPCH-567 could thus serve as diluting strain. Root treatment with mixed KMPCH/ $\mathrm{KMPCH}-567$ inocula with 1 and 10\% KMPCH resulted, at the end of an ISR experiment, in a relative $\mathrm{KMPCH}$ recovery from roots of, respectively, $1.14 \pm 0.33 \%$ and $9.21 \pm 1.09 \%$ (mean from three experiments \pm standard error [SE]). Because values for KMPCH recovery from roots were not significantly different from the initial value of $\mathrm{KMPCH}$ in the inoculum (student's $t$ test, $P=0.05$ ), it can be concluded that the $\mathrm{KMPCH} / \mathrm{KMPCH} 567$ ratio is stable throughout the experiment. With a total root density of $10^{8} \mathrm{CFU} \mathrm{g}^{-1}$ root in all treatments, root inoculation with 10,1 , and $0.1 \% \mathrm{KMPCH}$ thus resulted in a KMPCH density of, respectively, $10^{7}, 10^{6}$, and $10^{5} \mathrm{CFU} \mathrm{g}^{-1}$ root. KMPCH root density clearly affected ISR (Fig. 2): in three independent experiments ISR was always observed for treatments resulting in a KMPCH root density of $10^{8}$ or $10^{5} \mathrm{CFU} \mathrm{g} \mathrm{g}^{-1}$ root while either one or two intermediate KMPCH root densities did not result in ISR.

Now that it has been demonstrated that a KMPCH root density of $10^{5} \mathrm{CFU} \mathrm{\textrm {g } ^ { - 1 }}$ root, which is equivalent to the density observed in vivo, induces systemic resistance, the SA concentration (Fig. 1B) that corresponds to this root density can be investigated. In dual in vitro KMPCH- KMPCH-567 cultures with 100 to $0.1 \% \mathrm{KMPCH}$ and a stable ratio between the two

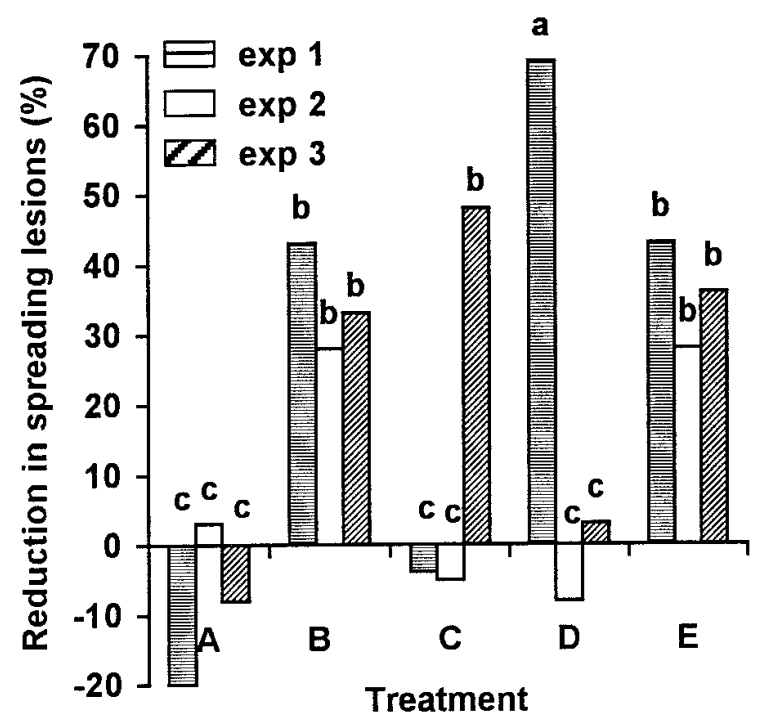

Fig. 2. Effect of root treatment with (A) KMPCH-567, (B) KMPCH, and mixed inocula containing (C) 10 , (D) 1 , and (E) $0.1 \% \mathrm{KMPCH}$ on the number of spreading Botrytis cinerea lesions on the first leaves of bean plants grown on perlite and a half-strength Hoagland solution. Leaves were inoculated with $1010-\mu \mathrm{l}$ drops of a $10^{6} \mathrm{~B}$. cinerea conidia $\mathrm{ml}^{-1}$

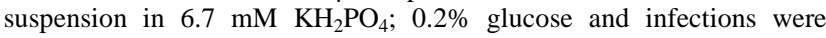
scored after 4 to 5 days. Results are presented as a reduction in spreading lesions relative to the number of spreading lesions in control plants. Data are from three independent experiments each with 10 leaves per treatment. Per experiment, bars with different letters are statistically different by logistic regression analysis at $P=0.05$. Bars marked with 'c' are not different from the control. strains (results not shown) the SA production by KMPCH was proportional to the amount of $\mathrm{KMPCH}$ in the culture (Table 1). Provided that the same proportionality exists in vivo the dilution series of KMPCH root density (Fig. 2) can be considered to be a dilution series of SA production. In this perspective, the similar absence of ISR for the 10 and $1 \% \mathrm{KMPCH}$ treatments (Fig. 2) and the $100 \mathrm{nM} \mathrm{SA}$ treatment (Fig. 1B) strongly suggests that the $0.1 \% \mathrm{KMPCH}$ treatment, which results in a KMPCH root density of $10^{5} \mathrm{CFU} \mathrm{\textrm {g } ^ { - 1 }}$ root, is equivalent to the 1 to $10 \mathrm{nM} \mathrm{SA}$ treatment. This is further supported by a theoretical calculation of the amount of SA produced by KMPCH on the roots. Provided that SA production by KMPCH in the perlite model is similar to the SA production in vitro and that SA production is proportional to the amount of KMPCH (Table 1) $10^{5} \mathrm{CFU}$ KMPCH should produce about $2 \mathrm{ng}$ of SA. This is similar to the amount of SA applied in the $1 \mathrm{nM} \mathrm{SA}$ solution $(90 \mathrm{ml}$ of $1 \mathrm{nM} \mathrm{SA} \approx 12.4 \mathrm{ng}$ of SA) and to the increase of $11.5 \mathrm{ng}$ of $\mathrm{SA} \mathrm{g}^{-1}$ root in the rhizosphere of soil-grown bean roots colonized by KMPCH (see below). Therefore, the treatment of roots with $1 \mathrm{nM} \mathrm{SA}$ seems to mimic best ISR by $\mathrm{KMPCH}$ in soil-grown plants. Most likely this conclusion also holds for ISR by 7NSK2 because the production in vitro of SA for this strain, about $6 \mu \mathrm{g}$ of SA $\mathrm{ml}^{-1}$ culture (De Meyer and Höfte 1997), is in the same order of magnitude as the production in vitro of SA by KMPCH.

\section{Root uptake of hydroponically fed SA.}

To study the mechanisms involved in SA-dependent ISR, 2week-old bean plants were hydroponically fed with SA solutions. Uptake was monitored by inclusion of $\left[{ }^{14} \mathrm{C}\right] \mathrm{SA}$ in 10 $\mu \mathrm{M}, 100 \mathrm{nM}$, and $5 \mathrm{nM}$ SA solutions and by measurement of radioactivity in roots at various time points during feeding of SA. In roots incubated with $5 \mathrm{nM}\left[{ }^{14} \mathrm{C}\right] \mathrm{SA}$, radioactivity increased gradually during the first $10 \mathrm{~h}$ of the treatment (Fig. 3 ), indicating that SA is taken up from the nutrient solution. From $10 \mathrm{~h}$ after the start of the incubation onward, the radioactivity in roots stabilized around $2.5 \mathrm{nCi}$ root system $^{-1}$, at least until $24 \mathrm{~h}$ after the start of the incubation (results not shown). An almost identical increase in radioactivity was observed for roots incubated with solutions containing $5 \mathrm{nM}$ $\left[{ }^{14} \mathrm{C}\right] \mathrm{SA}$ included in a total SA concentration of $100 \mathrm{nM}$ or 10 $\mu \mathrm{M}$ (results not shown). This demonstrates that, for the concentrations tested, the SA uptake is proportional to the SA concentration and that, consequently, the induction of resistance observed at different concentrations of SA (Fig. 1B) is not caused by a differential uptake of SA by the roots. In the same experiments, uptake of SA was also analyzed indirectly by multiplying the initial radioactivity in the solution $(0.32$

Table 1. Production of salicylic acid (SA) in dual cultures of Pseudomonas aeruginosa KMPCH and KMPCH-567 strains

\begin{tabular}{lcc}
\hline Proportion KMPCHa $^{\mathbf{a}}$ & Production of SA & Relative production \\
\hline 100 & $17,917 \pm 1,329^{\mathrm{b}}$ & $100^{\mathrm{c}}$ \\
10 & $2,033 \pm 602$ & 11.3 \\
1 & $210.1 \pm 44.2$ & 1.1 \\
0.1 & $45.2 \pm 1.9$ & 0.3 \\
\hline
\end{tabular}

a Percentage in the initial inoculum; the final concentration in the culture was about $2 \times 10^{9} \mathrm{CFU} \mathrm{ml}{ }^{-1}$.

${ }^{\mathrm{b}}$ Mean \pm standard error of three cultures in one representative experiment expressed as nanograms of SA ml${ }^{-1}$ culture.

${ }^{c}$ Relative to a pure KMPCH culture. 
$\mathrm{nCi} \mathrm{ml} \mathrm{m}^{-1}$ ) with the amount of nutrient solution taken up by the plant. As shown in Figure 3, these calculated values were consistently lower than the observed radioactivity in roots, especially from $4 \mathrm{~h}$ after the start of the incubation onward. This was also observed for $100 \mathrm{nM}$ or $10 \mu \mathrm{M}$ SA (results not shown) and suggests that, between 4 and $10 \mathrm{~h}$ after the start of the incubation, uptake of SA is more than directly proportional to concentration of SA in the solution.

After 1 and 2 days of hydroponic feeding with $10 \mu \mathrm{M}, 100$ $\mathrm{nM}$, and $1 \mathrm{nM}$ SA solutions, SA levels were also measured directly (Table 2). In roots fed with $10 \mu \mathrm{M}$ SA total SA levels increased by about $2 \mu \mathrm{g}$ of $\mathrm{SA} \mathrm{g}^{-1}$ root over basal levels, which is, in correlation with the above mentioned results, most likely due to SA uptake from the nutrient solution. Feeding with 100 and $1 \mathrm{nM}$ SA did not significantly affect total basal levels of SA in roots (Table 2). This is not surprising since, compared with the treatment with $10 \mu \mathrm{M} \mathrm{SA}$, a proportional SA uptake would correspond to only 20 and $0.2 \mathrm{ng}$ $\mathrm{g}^{-1}$ root, respectively, for feeding 100 and $1 \mathrm{nM} \mathrm{SA}$.

\section{PAL activity in roots during hydroponic SA feeding.}

Possible involvement of the phenylpropanoid pathway in SAdependent ISR was analyzed by the activity of the key enzyme PAL in roots. After $8 \mathrm{~h}$ of hydroponic feeding with $10 \mu \mathrm{M}, 100$

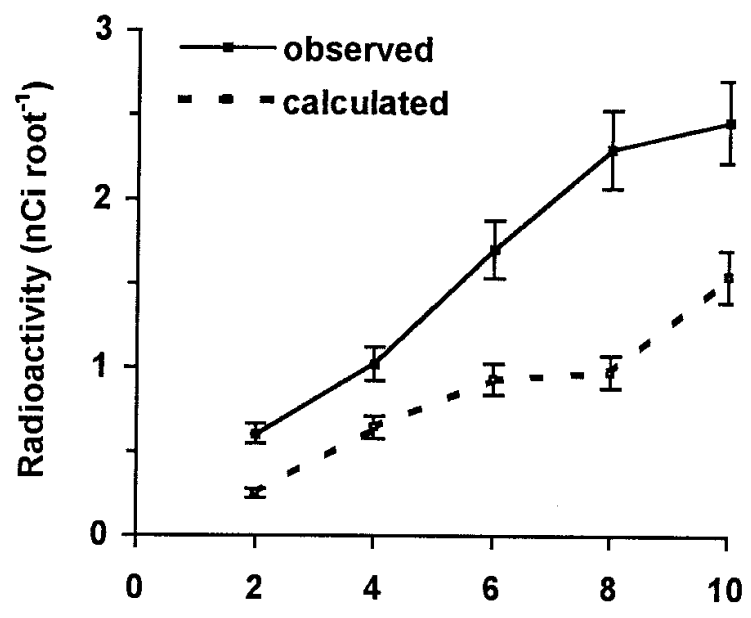

Time after transfer to SA solution (hours)

Fig. 3. Radioactivity in roots of plants root-incubated in a half-strength Hoagland solution containing $5 \mathrm{nM}\left[{ }^{14} \mathrm{C}\right]$ salicylic acid (SA). Measured values represent means for three plants \pm standard error of one representative experiment. For the same plants calculated values were obtained by multiplication of the volume of nutrient solution taken up by the plant with the radioactivity in the nutrient solution at the start of the experiment $\left(0.32 \mathrm{nCi} \mathrm{ml}^{-1}\right)$ and are represented \pm standard error.
$\mathrm{nM}$, or $1 \mathrm{nM}$ SA, PAL activity in roots did not differ from PAL activity in control plants (results not shown). However, after 24 $\mathrm{h}$, PAL activity in roots treated with $10 \mu \mathrm{M}$ or $1 \mathrm{nM}$ SA was significantly higher than in roots treated with $100 \mathrm{nM} \mathrm{SA}$ or plain nutrient solution (Fig. 4). Because only resistance-inducing SA concentrations (Fig. 1B) affected PAL activity, this phenomenon is probably linked to ISR. It is, however, unlikely that the increase in PAL activity caused a significant production of SA in roots because after $48 \mathrm{~h}$ of feeding at $1 \mathrm{nM}$ the amount of SA did not increase over basal levels (Table 2). After $48 \mathrm{~h}$, PAL activity in roots fed with $10 \mu \mathrm{M}$ or $1 \mathrm{nM}$ SA dropped again to levels observed in controls and roots fed with $100 \mathrm{nM}$ SA (results not shown). This does not necessarily indicate that feeding of $10 \mu \mathrm{M}$ or $1 \mathrm{nM}$ SA causes a transient induction of PAL in roots because after $48 \mathrm{~h}$ the SA concentration in the nutrient solution might have dropped to non-inducing levels.

SA levels in leaves after hydroponic feeding of SA.

After $24 \mathrm{~h}$ of feeding with $10 \mu \mathrm{M}, 100 \mathrm{nM}$, and $5 \mathrm{nM}$ SA solutions containing $5 \mathrm{nM}\left[{ }^{14} \mathrm{C}\right] \mathrm{SA}$, the radioactivity in first

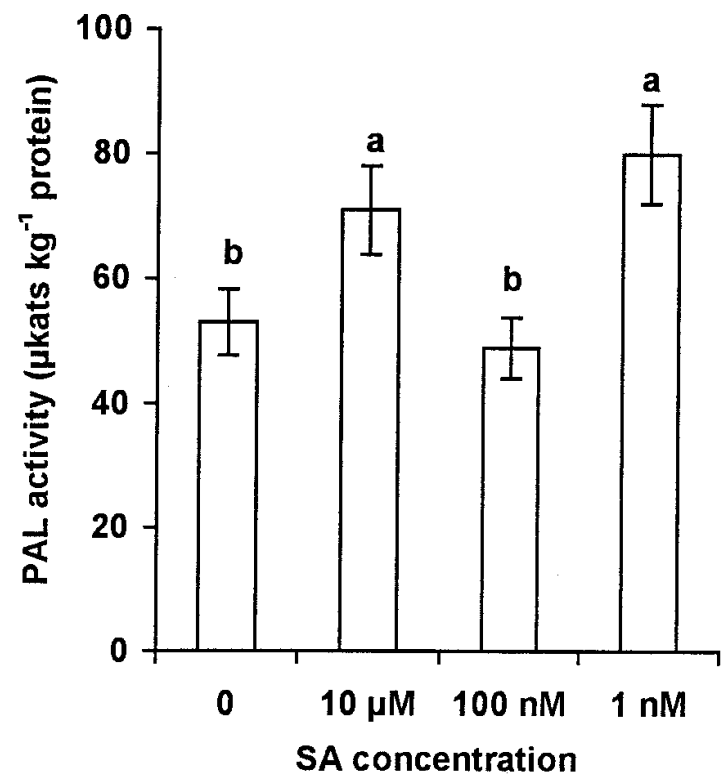

Fig. 4. Phenylalanine ammonia-lyase (PAL) activity in roots after $24 \mathrm{~h}$ incubation of plants in a half-strength Hoagland solution containing salicylic acid (SA). Data are means \pm standard error for four experiments each with five replications per treatment. Statistical analysis was performed on pooled data, as interaction between treatment and experiment was not significant at $P=0.05$ by analysis of variance. Treatment means were compared with Fisher's least significant difference test at $P=0.05$. Bars with different letters are statistically different.

Table 2. Time course of changes in free and bound salicylic acid (SA) in leaves and roots of bean plants hydroponically fed with different concentrations of SA

\begin{tabular}{|c|c|c|c|c|c|c|c|c|c|}
\hline \multirow[b]{2}{*}{ Organ } & \multirow{2}{*}{$\begin{array}{c}\text { Time }^{\mathrm{a}} \\
\text { (h) }\end{array}$} & \multicolumn{4}{|c|}{ Free SA } & \multicolumn{4}{|c|}{ Bound SA } \\
\hline & & Control & $10 \mu \mathrm{M}$ & $100 \mathrm{nM}$ & $1 \mathrm{nM}$ & Control & $10 \mu \mathrm{M}$ & $100 \mathrm{nM}$ & $1 \mathrm{nM}$ \\
\hline \multirow[t]{2}{*}{ Root } & 24 & $115.0 \pm 13.7^{b}$ & $114.2 \pm 17.0$ & $123.4 \pm 17.6$ & $90.41 \pm 6.1$ & $251.2 \pm 38.4$ & $2,265.9 \pm 560.7$ & $283.4 \pm 47.6$ & $207.9 \pm 25.1$ \\
\hline & 48 & $152.1 \pm 18.3$ & $148.6 \pm 36.7$ & $102.0 \pm 23.4$ & $115.5 \pm 6.0$ & $229.0 \pm 69.3$ & $2,493.2 \pm 290.4$ & $172.9 \pm 28.7$ & $237.7 \pm 69.1$ \\
\hline \multirow[t]{2}{*}{ Leaf } & 24 & $37.3 \pm 2.3$ & $55.1 \pm 3.8$ & $36.9 \pm 3.2$ & $37.1 \pm 3.6$ & $225.5 \pm 36.0$ & $216.3 \pm 40.3$ & $238.3 \pm 40.1$ & $217.6 \pm 54.3$ \\
\hline & 48 & $45.4 \pm 4.8$ & $113.1 \pm 20.6$ & $47.0 \pm 4.5$ & $114.9 \pm 18.1$ & $160.8 \pm 20.1$ & $185.2 \pm 34.6$ & $172.6 \pm 35.0$ & $167.5 \pm 29.2$ \\
\hline
\end{tabular}

a Time of hydroponic feeding.

${ }^{\mathrm{b}}$ Mean \pm standard error of two experiments each with three replicates per treatment, expressed in $\mathrm{ng} \mathrm{g}^{-1}$ fresh weight. 
leaves had increased by $0.010 \pm 0.002 \mathrm{nCi} \mathrm{g}^{-1}$ leaf (mean \pm $\mathrm{SE}$ ) over control levels for all treatments mentioned. This indicates that, for the concentrations tested, transport of SA fed to roots to first leaves is proportional to the concentration of the SA in the applied solution. However, comparing these values with radioactivity levels in roots (Fig. 3), it should be stressed that the vast majority of the SA taken up from the nutrient solution remains confined to the roots. For the $10 \mu \mathrm{M}$ treatment the increase of $0.010 \mathrm{nCi} \mathrm{g}^{-1}$ leaf is equivalent to 31 $\mu \mathrm{l}$ of nutrient solution (nutrient solution $\approx 0.32 \mathrm{nCi} \mathrm{ml}^{-1}$ ) or 43 ng of SA $\left(10 \mu \mathrm{M} \approx 1.38 \mu \mathrm{g} \mathrm{m}^{-1}\right)$. Because a similar increase in free SA levels, about $18 \mathrm{ng} \mathrm{g}^{-1}$ leaf, was observed in leaves from plants incubated in $10 \mu \mathrm{M}$ SA (Table 2), it can be concluded that the increase in free SA in leaves is due to transport of SA from the nutrient solution. Similar reasoning demonstrates that for feeding 100 or $1 \mathrm{nM} \mathrm{SA}$, an increase in SA proportional to the increase found for treatment with $10 \mu \mathrm{M}$ SA will not affect basal SA levels in leaves. Measurements of SA in leaves $24 \mathrm{~h}$ after treatment with 100 or $1 \mathrm{nM}$ confirm this statement (Table 2). After $48 \mathrm{~h}$ of feeding, free SA levels in leaves from plants treated with $10 \mu \mathrm{M}$ and $1 \mathrm{nM}$ had significantly increased above basal levels (Table 2). Because free SA levels were unaffected after treatment with $100 \mathrm{nM} \mathrm{SA}$ or plain nutrient solution this phenomenon is associated with ISR. For the treatment at $1 \mathrm{nM}$ it is physically impossible that the increase in SA in leaves results from uptake of SA from the nutrient solution because $20 \mathrm{ml}$ of $1 \mathrm{nM}$ SA merely contains a total of $3 \mathrm{ng}$ of SA. Consequently, this increase in SA has to result from de novo synthesis. Most likely this is also the case for the treatment at $10 \mu \mathrm{M}$ because transport of SA to leaves is very low after $24 \mathrm{~h}$ of feeding SA (see above). However, our data do not exclude the possibility that after $48 \mathrm{~h}$ the increased free SA levels in leaves of plants treated with $10 \mu \mathrm{M}$ SA result from the transport of SA from the nutrient solution to the leaves.

\section{PAL activity and SA levels in soil-grown plants root colonized by $P$. aeruginos $a$ strains and in vivo production of SA by $P$. aeruginosa.}

To compare feeding of $1 \mathrm{nM}$ SA to root colonization by $P$. aeruginosa strains that produce SA we used root bacterized soil-grown plants that were 3 weeks old, the age at which we routinely assay for ISR (De Meyer and Höfte 1997). PAL activity in roots of control plants was $22.65 \pm 1.01 \mu \mathrm{kats}_{\mathrm{kg}}{ }^{-1}$ protein (mean of two experiments $\pm \mathrm{SE}$ ), normally distributed and not statistically different from PAL activity in roots treated with the SA-deficient and ISR $^{-}$strain MPFM1-569, which was $22.57 \pm 1.60 \mu \mathrm{kats}_{\mathrm{kg}}^{-1}$ protein. In roots treated with the systemic resistance-inducing strain $\mathrm{KMPCH}, \mathrm{PAL}$ activity was not normally distributed. Most roots had a PAL activity similar to that of control roots but $50 \%$ (6 out of 12) had PAL activity of more than $28.61 \mu \mathrm{kats} \mathrm{kg}^{-1}$ protein, which corresponds to the mean of control $+2 \times$ standard deviation (SD; Fig. 5). If PAL activity in KMPCH-treated plants followed the same normal distribution as in control plants only $5 \%$ of the values should fall outside of the mean $\pm 2 \times$ SD interval. It can thus be concluded that colonization of roots with $P$. aeruginosa $\mathrm{KMPCH}$ increased PAL activity in roots, but not in all plants. This differential induction was not linked to differences in $\mathrm{KMPCH}$ root density because $\mathrm{KMPCH}$ root density was 2 to $4 \times 10^{5} \mathrm{CFU} \mathrm{g}^{-1}$ root for all plants. In addi- tion, the expression of ISR in plants that did not show an increase in PAL activity in roots (results not shown) suggests that the increase in PAL activity in roots of 3-week-old plants is not essential for ISR. In leaves, free SA levels in plants treated with KMPCH were significantly higher than in control plants or plants treated with MPFM1-569 (increase of about $50 \%$ ) but amounts of bound SA were similar for all treatments (Table 3). In vivo production of SA by KMPCH was measured in the rhizosphere of soil-grown bean plants. In one representative experiment we detected $17.9 \pm 2.9 \mathrm{ng}$ of $\mathrm{SA} \mathrm{g}^{-1}$ root (mean $\pm \mathrm{SE}$ ) in a rhizosphere extract of control plants. In the rhizosphere of plants colonized by $\mathrm{KMPCH}$ this value was increased by $11.5 \pm 1.8 \mathrm{ng} \mathrm{SA} \mathrm{g}^{-1}$ root.

\section{DISCUSSION}

In this study we used a model with bean plants grown on perlite and nutrient solution to analyze molecular changes associated with ISR by $P$. aeruginosa 7NSK2. Just like in soilgrown plants (De Meyer and Höfte 1997) ISR by 7NSK2 depended on the production of SA by 7NSK2 (Fig. 1A). The

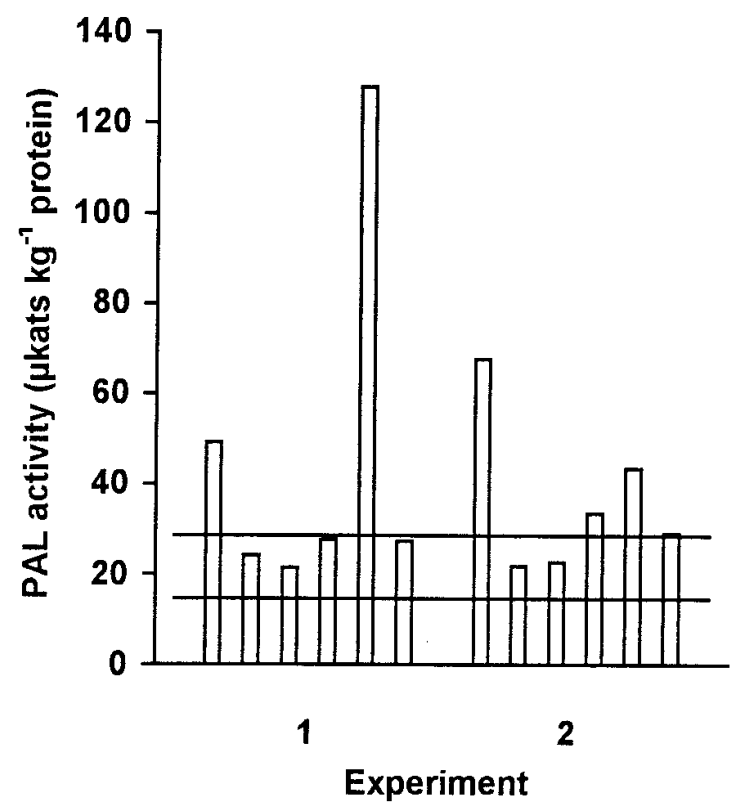

Fig. 5. Phenylalanine ammonia-lyase (PAL) activity in roots of 3-weekold, soil-grown bean plants treated with Pseudomonas aeruginosa $\mathrm{KMPCH}$. Bars represent values from single plants from two independent experiments. Lines indicate the $95 \%$ confidence interval for PAL activity in control plants (mean $\pm 2 \times$ standard deviation), pooled for the same two experiments.

Table 3. Levels of free and bound salicylic acid (SA) in leaves of 3week-old soil-grown plants treated with Pseudomonas aeruginosa strains

\begin{tabular}{lcc}
\hline Treatment & Free SA & Bound SA \\
\hline Control & $39.7 \pm 4.5^{\mathrm{a}}$ & $125.0 \pm 15.9$ \\
KMPCH & $59.2 \pm 7.1$ & $142.9 \pm 20.4$ \\
MPFM1-569 & $37.8 \pm 5.2$ & $115.6 \pm 14.3$ \\
\hline
\end{tabular}

\footnotetext{
${ }^{a}$ Mean \pm standard error of two experiments each with three replicates
} per treatment, expressed in $\mathrm{ng} \mathrm{g}^{-1}$ fresh weight. 
amount of SA involved in ISR by 7NSK2 was indirectly estimated in experiments with the 7NSK2 mutant KMPCH. Analysis of ISR by a dilution series of SA-containing nutrient solutions and by a dilution series in KMPCH root density revealed that both series included intermediate doses that did not induce resistance (Figs. 1B and 2). In a similar dilution series of KMPCH density in vitro, production of SA was proportional to the amount of KMPCH (Table 1). Therefore, the dilution series of SA in the nutrient solution and of $\mathrm{KMPCH}$ root density represent the same phenomenon. Matching of both series strongly suggests that a KMPCH root density of about $10^{5} \mathrm{CFU} \mathrm{\textrm {g } ^ { - 1 }}$ root, which is similar to the colonization of soil-grown plants (De Meyer and Höfte 1997), is equivalent to root treatment with 1 to $10 \mathrm{nM}$ SA. Because the theoretical amount of SA produced by $10^{5} \mathrm{CFU}$ can be estimated at $2 \mathrm{ng}$ based on an SA production proportional to the amount of $\mathrm{KMPCH}$, and because the amount of SA applied via the $1 \mathrm{nM}$ SA solution is $12.4 \mathrm{ng}$, it was concluded that feeding $1 \mathrm{nM}$ is the most realistic representation of the situation in the soil. This is consistent with the increase of $11.5 \mathrm{ng}$ of SA g${ }^{-1}$ root over control levels in the rhizosphere of soil-grown bean plants colonized by KMPCH. Because SA production by 7NSK2 is in the same order of magnitude as for KMPCH these conclusions are most likely also valid for ISR by 7NSK2.

To investigate whether the SAR pathway interferes in ISR by $P$. aeruginosa 7NSK2 we investigated biochemical changes upon hydroponic feeding with the equivalent SA concentration of $1 \mathrm{nM}$. After $48 \mathrm{~h}$, the nanogram amounts of SA applied by this treatment resulted in an increase in leaf free SA from basal levels of 45.4 to $114.9 \mathrm{ng} \mathrm{g}^{-1}$ leaf (Table 2). Although an increase in leaf free SA is typical for SAR the increase observed here is lower than in the case of full SAR and seems insufficient for the induction of pathogenesis-related (PR) proteins (see below) that is a typical characteristic of SAR. This suggests that $1 \mathrm{nM}$ SA feeding only results in a partial induction of the SAR signal transduction pathway. Because the amount of SA applied by feeding $1 \mathrm{nM} \mathrm{SA}$ is significantly lower than the increase in free SA in the leaves, systemic transport of the applied SA cannot explain the increase in free SA in the leaves. Most likely the induction of PAL activity in roots (Fig. 4) is the link between the applied SA and the induction of the SAR signal transduction pathway. This is supported by the timing of the induction of PAL, which occurs after $24 \mathrm{~h}$ while the increase in free SA is only observed after $48 \mathrm{~h}$. Moreover, this is consistent with the plausible role for PAL in the generation of the long-distance signal for SAR in cucumber (Smith-Becker et al. 1998). The activation of the SAR pathway in ISR by KMPCH was confirmed by analysis of PAL activity in roots and SA levels in leaves of 3-week-old soil-grown plants. PAL activity in roots colonized by the ISRinducing and SA-producing strain $\mathrm{KMPCH}$ increased over control levels in $50 \%$ of the plants tested (Fig. 5) while roots colonized by the SA and ISR negative strain MPFM1-569 had the same PAL activity as control plants. This confirms that small amounts of SA produced by KMPCH induce PAL but also suggests that PAL induction is not continuous. Because induction of PAL is most likely associated with the generation of the SAR signal, its absence in some KMPCH-treated plants may indicate that resistance was already induced rather than that resistance was not induced. This is consistent with the observation of ISR in plants that did not show an increased activity of PAL in the roots (results not shown). More consistent over all plants was the $50 \%$ increase in free SA over control levels in leaves of KMPCH-treated plants (Table 3) although the increase in free SA in the leaves was less pronounced than after 2 days of feeding $1 \mathrm{nM}$ SA (Table 2). Possibly, this can be explained by the different timing of the SA measurement with respect to the induction stimulus. Based on these results, we conclude that nanogram amounts of SA produced by the rhizobacterium $P$. aeruginosa 7 NSK2 activate the SAR pathway. However, the induction might be only partial because the increase in free SA in leaves is lower than in the case of full SAR.

This conclusion is consistent with the observation that in the tobacco-TMV model ISR by 7NSK2 depends, just like SAR induced by TMV (Vernooij et al. 1994), on in planta accumulation of SA for its expression but not for its induction (G. De Meyer, K. Audenaert, and M. Höfte, unpublished). In addition, the absence of expression of PR1a in tobacco plants treated with $P$. aeruginosa 7NSK2 (G. De Meyer, K. Audenaert, and M. Höfte, unpublished) further suggests that activation of the SAR pathway by $P$. aeruginosa 7NSK2 might only occur partially. Most likely the induction of the SAR pathway by nanogram amounts of SA is also involved in ISR by other rhizobacteria that depend on SA for the induction of systemic resistance. Maurhofer et al. (1998) detected a similar increase of $45.3 \mathrm{ng}$ of SA plant ${ }^{-1}$ in the rhizosphere of tobacco plants whose roots were colonized by $P$. fluorescens $\mathrm{CHA} 0$, a strain that produces SA and induces systemic resistance. Moreover, Maurhofer et al. (1994) detected an increase in levels of free SA in leaves of tobacco plants colonized by $P$. fluorescens $\mathrm{CHA} 0$ on the roots that was similar to the increase in free SA in leaves of bean plants treated with $1 \mathrm{nM}$ SA or colonized by KMPCH on the roots.

Plants grown in nutrient solutions containing 1 to $100 \mu \mathrm{M}$ SA showed an enhanced resistance to $B$. cinerea that was similar to the enhanced resistance in the $1 \mathrm{nM}$ SA treatment (Fig. 1B). Similar experiments in the tobacco-TMV model showed that feeding $100 \mu \mathrm{M}$ SA induced resistance to TMV, increased levels of SA by several $\mu \mathrm{g} \mathrm{g}^{-1}$ leaf (Enyedi et al. 1992), and induced expression of PR1a (Mur et al. 1996), all typical characteristics of SAR. Hydroponic feeding with 25 $\mu \mathrm{M}$ SA induced nearly the same resistance to TMV as feeding with $100 \mu \mathrm{M}$ SA but only increased free SA levels from 200 to $550 \mathrm{ng} \mathrm{g}^{-1}$ leaf (Enyedi et al. 1992). Feeding of $10 \mu \mathrm{M}$ SA did not directly induce PR1a, but only potentiated PR1a expression upon TMV infection (Mur et al. 1996). In this perspective it is unlikely that feeding $10 \mu \mathrm{M}$ SA to bean, associated with an increase in free SA from 45.4 to $113.1 \mathrm{ng} \mathrm{g}^{-1}$ leaf (Table 2), will induce expression of PR proteins. Possibly, this modest increase in free SA in the leaves results in the potentiation of PR-protein expression upon pathogen infection, which then leads to an enhanced resistance. Alternatively, a so far unidentified defense response might be involved. Our data do not exclude that the increase in free SA in leaves after feeding with $10 \mu \mathrm{M}$ SA results from systemic transport of the applied SA to the leaves. However, the strong parallels with the $1 \mathrm{nM}$ SA treatment on the levels of induced resistance (Fig. 1), accumulation of free SA (Table 2), and induction of PAL (Fig. 4) suggest that the increase in free SA in the leaves of plants fed with $10 \mu \mathrm{M}$ results from de novo synthesis. This would also explain why the major increase in free SA in the leaves is only 
observed after the induction of PAL in the roots. Therefore, we propose that the systemic increase in free SA in leaves in the $10 \mu \mathrm{M}$ SA treatment results from the activation of SAR signaling. In this perspective the application of $1 \mathrm{nM}$ SA to the roots has the same effect as $10 \mu \mathrm{M}$. This is consistent with the finding of Vernooij et al. (1994) that the systemic signal for SAR can be fully induced from NahG tissue that does not allow significant accumulation of SA and that this signal is induced before significant SA accumulation in cucumber (Smith-Becker et al. 1998). An effect of nanogram amounts of SA in tissues with a significantly higher basal level of SA is only possible in the case of compartmentalization of SA in plant cells or in specific cell types. In this way, specific cell sites or cell types with low basal SA levels could respond to increases of SA in the nanogram range. The observation that SA uptake is more than directly proportional to concentration of SA (Fig. 3), and the active uptake of SA by bean roots (Schulz et al. 1993), both indicate phloem loading. Therefore, we speculate that the applied SA is rapidly loaded in phloem cells, which are involved in the generation of the systemic signal for SAR (Smith-Becker et al. 1998) and might have a high sensitivity to SA. In addition, an effect of nanogram amounts of SA in tissues with a significantly higher basal level of SA is also impossible without a control mechanism that prevents the triggering of the SAR pathway by basal levels of SA. This can explain why no ISR is observed after treatment with $100 \mathrm{nM} \mathrm{SA}$, a concentration that corresponds to an amount of SA that is close to the basal level of SA in plants. Possibly, this control mechanism involves the sequestration of the applied SA following glucosylation (Enyedi and Raskin, 1993) and might be overwhelmed after application of micromolar amounts of SA.

\section{MATERIALS AND METHODS}

\section{Bacterial strains, culture, and root colonization.}

Phenotypes of $P$. aeruginosa strains used in this study are represented in Table 4. KMPCH-567 was derived from $\mathrm{KMPCH}$ by gene replacement, as previously described (De Meyer and Höfte 1997), with the suicide plasmid pME3356, which carries a mutated copy of the $p c h A$ gene, essential for SA synthesis in P. aeruginosa (Serino et al. 1995). The SAdeficient phenotype of KMPCH-567 was confirmed by thin layer chromatography of an ethyl acetate extract from an ironlimited culture, as previously described (De Meyer and Höfte 1997). Bacteria were routinely grown overnight at $37^{\circ} \mathrm{C}$ on King's medium B (KB; King et al. 1954), scraped off the plates, and suspended in sterile saline $(0.85 \% \mathrm{NaCl})$. This suspension was diluted to the desired bacterial concentration based on $\mathrm{OD}_{595}$. For the analysis of SA production in vitro, bacteria were grown in casamino acids medium as previously described (De Meyer and Höfte 1997). Root colonization by
7NSK2 or derived mutants was determined by dilution plating of a macerated root sample on $\mathrm{KB}$ and colony counting after a selective $14 \mathrm{~h}$ incubation at $37^{\circ} \mathrm{C}$. For roots inoculated with mixed KMPCH/KMPCH-567 solutions the relative amount of both strains was determined by transfer of the colonies obtained to new $\mathrm{KB}$ plates. After a $2 \mathrm{~h}$ pre-incubation at $37^{\circ} \mathrm{C}$ these plates were sprayed with a $10^{8} \mathrm{CFU} \mathrm{ml^{-1 }}$ MPFM1-569 suspension and further incubated at $37^{\circ} \mathrm{C}$ for $12 \mathrm{~h}$. UV exposure then revealed a blue halo around KMPCH colonies because MPFM1-569 had converted the SA produced by $\mathrm{KMPCH}$ to the blue fluorescent pyochelin. This was not observed for KMPCH-567 colonies because the latter strain does not produce SA. With this technique we determined the $\mathrm{KMPCH} / \mathrm{KMPCH}-567$ ratio of five plants from the 10 and $1 \%$ $\mathrm{KMPCH}$ treatments by analyzing, respectively, 50 and 200 colonies per plant. Per treatment the obtained KMPCH/ KMPCH-567 ratios of three experiments were pooled and compared with the initial ratio by student's $t$ test $(P=0.05)$.

\section{Plant growth.}

For plant growth on perlite and nutrient solution, beans (Phaseolus vulgaris cv. Prelude; Royal Sluis, Enkhuizen, The Netherlands) were surface disinfected with $0.1 \% \mathrm{HgCl}_{2}$ for 7 min, rinsed three times with sterile, demineralized water (SDW) and germinated in autoclaved quartz sand mixed with SDW. Roots of 1-week-old seedlings were rinsed in SDW to remove most of the sand and transferred to sterile $20-\mathrm{cm}$ test tubes $(3 \mathrm{~cm}$ diameter) that were subsequently filled with autoclaved perlite, and $40 \mathrm{ml}$ of a half-strength Hoagland solution (Hoagland and Arnon 1938). Tubes were covered with aluminum foil and transferred to a growth chamber. Seven and 10 days after transfer, another $25 \mathrm{ml}$ of the half-strength Hoagland solution was added to each tube. In this model SA was applied to the plants by including the desired concentration in the nutrient solution for both the initial and supplementary applications. Root colonization by $P$. aeruginosa strains was achieved by dipping the roots in a $10^{8} \mathrm{CFU} \mathrm{ml}{ }^{-1}$ bacterial suspension prior to transfer to tubes. For hydroponic SA-feeding experiments, plants were grown for 1 week in tubes with plain nutrient solution, removed from the tubes, cleared from perlite, and placed in 50-ml conical flasks with $20 \mathrm{ml}$ of a halfstrength Hoagland solution amended with SA at the desired concentrations. Plants were then retransferred to the growth chamber.

In experiments with soil-grown plants $P$. aeruginosa root colonization was achieved by a combined seed and soil treatment and plants were grown under greenhouse conditions for 3 weeks (De Meyer and Höfte 1997).

\section{Assay for ISR to B. cinerea.}

First leaves of the bean plants that had grown on perlite and nutrient solution for 2 weeks were excised by cutting the peti-

Table 4. Pseudomonas aeruginosa 7NSK2 and derived mutants used in this study

\begin{tabular}{llll}
\hline Strain & Relevant characteristics & Reference or source & \\
\hline 7NSK2 & Wild type, $\mathrm{Pvd}^{+}, \mathrm{Pch}^{+}, \mathrm{SA}^{+}$ & Iswandi et al. 1987 \\
KMPCH & $\mathrm{Pvd}^{-}, \mathrm{Pch}^{-}, \mathrm{SA}^{+}$chemical mutant of MPFM1 $\left(\mathrm{Pvd}^{-}, \mathrm{Pch}^{+}, \mathrm{SA}^{+}\right.$, obtained by Tn 5 mutagenesis of 7NSK2) & Höfte et al. 1993 \\
MPFM1-569 & $\mathrm{Pvd}^{-}, \mathrm{Pch}^{-}, \mathrm{SA}^{-} ; p c h$ A replacement mutant of MPFM1; able to convert SA to Pch & De Meyer and Höfte 1997 \\
KMPCH-567 & $\mathrm{Pvd}^{-}, \mathrm{Pch}^{-}, \mathrm{SA}^{-}, p c h A$ replacement mutant of KMPCH; unable to convert SA to Pch & This work & \\
\hline
\end{tabular}

a Abbreviations: Pvd, pyoverdin; Pch, pyochelin; and SA, salicylic acid. 
ole near the stem. The petiole was immediately wrapped in wet absorbing paper. Leaves were transferred to trays and placed on a plastic lattice supported by glass rods. The wrapped petioles were put through the lattice in order to touch several layers of wet absorbing paper on the bottom of the tray. Leaves were then inoculated with $1010-\mu$ drops of a $10^{6}$ B. cinerea spores $\mathrm{ml}^{-1}$ suspension, obtained as previously described (De Meyer and Höfte 1997) in $6.7 \mathrm{mM} \mathrm{KH_{2 }} \mathrm{PO}_{4}$ and $0.2 \%$ glucose. Trays were covered with transparent plastic foil and incubated at $20^{\circ} \mathrm{C}$. Four to 5 days after inoculation, B. cinerea infections were scored as a spreading lesion if the fungus had developed beyond the inoculum drop of about $5 \mathrm{~mm}$ diameter. Per treatment, 10 leaves, taken from 10 different plants, were inoculated. Results were categorized in a dichotomous variable (spreading or nonspreading) and analyzed by logistic regression analysis. Data for experiments with the same setup were pooled for statistical analysis if interaction between treatment and experiment was not significant at $P=0.05$.

\section{Determination of PAL activity.}

PAL activity was determined as described by Edwards and Kessmann (1992). Roots were ground to a fine powder in $\mathrm{N}_{2}$ and extracted with $50 \mathrm{mM}$ Tris- $\mathrm{HCl}(\mathrm{pH} \mathrm{8.5)}$ containing 14 $\mathrm{mM}$ 2-mercaptoethanol and 5\% polyvinylpyrrolidone. Homogenates were centrifuged $10 \mathrm{~min}$ at $8,000 \times g$ and the resulting supernatant was used for PAL activity and protein determination. Protein levels were determined with a protein assay kit (Bio-Rad, Hercules, CA) and bovine serum albumin (Sigma, St. Louis, MO) as a standard. PAL activity was determined at $40^{\circ} \mathrm{C}$ in $1 \mathrm{ml}$ of $50 \mathrm{mM}$ Tris- $\mathrm{HCl}(\mathrm{pH} 8.5) 10 \mathrm{mM}$ phenylalanine (Sigma). During $1 \mathrm{~h}$, the increase in $\mathrm{OD}_{290}$, indicating the conversion of phenylalanine to trans cinnamic acid was monitored with a spectrophotometer (Uvikon 922; Kontron, B.R.S., Anderlecht, Belgium). Per treatment and time point, four plants were analyzed and the experiment was performed three times. Statistical analysis was performed by analysis of variance on pooled data from all experiments because interaction between treatment and experiment was not significant. Means were compared with Fisher's least significant difference (LSD) test. For soil-grown plants, PAL activity was determined for six plants per treatment. The experiment was performed twice and treatments were compared by student's $t$ test at $P=0.05$ on pooled data from both experiments.

\section{Quantification of SA in plant tissue, in the rhizosphere, and in bacterial cultures.}

Free and bound SA in plant tissue was quantified as described by Meuwly and Métraux (1993) with o-anisic acid as an internal standard to correct for extraction efficiency. Samples were taken from roots and leaves of three plants per treatment and time point and experiments were performed twice. For rhizosphere extraction three samples of $10 \mathrm{~g}$ of root pooled from different plants were analyzed per treatment. Samples were extracted as described by Maurhofer et al. (1998) after addition of o-anisic acid to correct for extraction efficiency. Bacterial cultures were extracted in triplicate per culture as described previously (De Meyer and Höfte 1997). However, o-anisic acid was added to the culture prior to the extraction to correct for extraction efficiency and SA was quantified by high-pressure liquid chromatography as described by Meuwly and Métraux (1993).

\section{Uptake of hydoponically fed $\left[{ }^{14} \mathrm{C}\right] \mathrm{SA}$.}

To monitor SA uptake, bean plants were hydroponically fed with nutrient solutions containing $5 \mathrm{nM}$ [carboxyl- ${ }^{14} \mathrm{C}$ ]SA (ICN, Irvine, CA; $56 \mathrm{mCi} / \mathrm{mmol}$ ). The solution was either applied as such or supplemented with nonlabeled SA to a final concentration of $100 \mathrm{nM}$ or $10 \mu \mathrm{M}$ SA. Every $2 \mathrm{~h}$, during the first $10 \mathrm{~h}$ of hydroponic feeding, leaves and roots of three plants per treatment were frozen and stored at $-20^{\circ} \mathrm{C}$. At the same time the remaining amount of nutrient solution was analyzed. Frozen material was lyophilized and combusted in a Biological Material Oxidizer Model 306 (Canberra-Packard, Zellik, Belgium). The combusted gases containing $\left[{ }^{14} \mathrm{C}\right]$ dioxide were collected in $8 \mathrm{ml}$ of Carbosorb I (Packard) and $10 \mathrm{ml}$ of Permafluor E+ (Packard). Radioactivity was determined by a liquid scintillation counter (Betamatic Basic, Kontron, B.R.S., Anderlecht, Belgium) and corrected for quenching based on an external standard ratio. Results were corrected for background radioactivity with values from untreated control plants. The experiment was performed twice.

\section{ACKNOWLEDGMENTS}

This work was supported by a grant from the Fund for Scientific Research-Flanders (FWO, Belgium) and by a specialization fellowship of the Flemish Institute for the stimulation of Scientific-Technological Research in Industry (IWT, Belgium) that was given to G. D. M. and K. Audenaert. We also thank G. Smagghe for advice on $\left[{ }^{14} \mathrm{C}\right]$ techniques.

\section{LITERATURE CITED}

Buysens, S., Heungens, K., Poppe, J., and Höfte, M. 1996. Involvement of pyochelin and pyoverdin in suppression of Pythium-induced damping-off of tomato by Pseudomonas aeruginosa 7NSK2. Appl. Environ. Microbiol. 62:865-871.

De Meyer, G., and Höfte, M. 1997. Salicylic acid produced by the rhizobacterium Pseudomonas aeruginosa 7NSK2 induces resistance to leaf infection by Botrytis cinerea on bean. Phytopathology 87:588593.

Edwards, R., and Kessmann, H 1992. Isoflavonoid phytoalexins and their biosynthetic enzymes. Pages 45-62 in: Molecular Plant Pathology: A Practical Approach. Vol. 2. S. Gurr, M. McPherson, and D. Bowles, eds. Oxford University Press, Oxford.

Enyedi, A. J., and Raskin, I. 1993. Induction of UDP-glucose: Salicylic acid glucosyltransferase activity in tobacco mosaic virus-inoculated tobacco (Nicotiana tabacum) leaves. Plant Physiol. 101:1375-1380.

Enyedi, A. J., Yalpani, N., Silverman, P., and Raskin, I. 1992. Localization, conjugation, and function of salicylic acid in tobacco during the hypersensitive reaction to tobacco mosaic virus. Proc. Natl. Acad. Sci. USA 89:2480-2484.

Hoagland, D. R., and Arnon, D. I. 1938. The water culture method for growing plants without soil. Calif. Agric. Exp. Stn. Bull. 347.

Höfte, M., Buysens, S., Koedam, N., and Cornelis, P. 1993. Zinc affects siderophore-mediated high affinity iron uptake systems in the rhizosphere Pseudomonas aeruginosa 7NSK2. Biometals 6:85-91.

Iswandi, A., Bossier, P., Vandenabeele, J., and Verstraete, W. 1987. Effect of seed inoculation with the rhizopseudomonas strain 7NSK2 on the root microbiota of maize (Zea mays) and barley (Hordeum vulgare). Biol. Fertil. Soils 3:153-158.

King, E. O., Ward, M., and Raney, D. E. 1954. Two simple media for demonstration of pyocyanin and fluorescin. J. Lab. Clin. Med. 44: 301-307.

Kloepper, J. W., Tuzun, S., and Kuc, J. A. 1992. Proposed definitions related to induced disease resistance. Biocontrol Sci. Technol. 2:349351.

Leeman, M., den Ouden, F. M., van Pelt, J. A., Dirkx, F. P. M., Steijl, H., Bakker, P. A. H. M., and Schippers, B. 1996. Iron availability affects induction of systemic resistance to Fusarium wilt of radish by $\mathrm{Pseu}$ domonas fluorescens. Phytopathology 86:149-155. 
Malamy, J., Carr, J. P., Klessig, D. F., and Raskin, I. 1990. Salicylic acid: A likely endogenous signal in the resistance response of tobacco to viral infection. Science 250:1002-1004.

Maurhofer, M., Hase, C., Meuwly, P., Métraux, J.-P., and Défago, G. 1994. Induction of systemic resistance of tobacco to tobacco necrosis virus by the root-colonizing Pseudomonas fluorescens strain CHA0: Influence of the gacA gene and of pyoverdine production. Phytopathology 84:139-146.

Maurhofer, M., Reimmann, C., Schmidli-Sacherer, P., Heeb, S., Haas, D., and Défago, G. 1998. Salicylic acid biosynthetic genes expressed in Pseudomonas fluorescens P3 improve the induction of systemic resistance in tobacco against tobacco necrosis virus. Phytopathology 88:678-684.

Meuwly, P., and Métraux, J.-P. 1993. Ortho-anisic acid as internal standard for the simultaneous quantitation of salicylic acid and its putative biosynthetic precursors in cucumber leaves. Anal. Biochem. 214:500-505.

Métraux, J.-P., Signer, H., Ryals, J., Ward, E., Wyss-Benz, M., Gaudin, J., Raschdorf, K., Schmid, E., Blum, W., and Inverardi, B. 1990. Increase in salicylic acid at the onset of systemic acquired resistance in cucumber. Science 250:1004-1006.

Mölders, W., Buchala, A., and Métraux, J.-P. 1996. Transport of salicylic acid in tobacco necrosis virus-infected cucumber plants. Plant Physiol. 112:787-792.

Mur, L. A. J., Naylor, G., Warner, S. A. J., Sugars, J. M., White, R. F., and Draper, J. 1996. Salicylic acid potentiates defence gene expression in tissue exhibiting acquired resistance to pathogen attack. Plant J. 9:559-571.

Pallas, J., Paiva, N., Lamb, C., and Dixon, R. 1996. Tobacco plants epigenetically suppressed in phenylalanine ammonia-lyase expression do not develop systemic acquired resistance in response to infection by tobacco mosaic virus. Plant J. 10:281-293.

Pieterse, C. M. J., van Wees, S. C. M., Hoffland, E., van Pelt, J. A., and van Loon, L. C. 1996. Systemic resistance in Arabidopsis induced by biocontrol bacteria is independent of salicylic acid accumulation and pathogenesis-related gene expression. Plant Cell 8:1225-1237.

Press, C. M., Wilson, M., Tuzun, S., and Kloepper, J. W. 1997. Salicylic acid produced by Serratia marcescens $90-166$ is not the primary determinant of induced systemic resistance in cucumber or tobacco. Mol. Plant-Microbe Interact. 10:761-768.

Ryals, J. A., Neuenschwander, U. H., Willits, M. G., Molina, A., Steiner, H.-Y., and Hunt, M. D. 1996. Systemic acquired resistance. Plant Cell 8:1809-1819.

Schulz, M., Schnabl, H., Manthe, B., Schweinhofen, B., and Casser, I. 1993. Uptake and detoxification of salicylic acid by Vicia faba and Fagopyrum esculentum. Phytochemistry 33:291-294.

Serino, L., Reimmann, C., Baur, H., Beyeler, M., Visca, P., and Haas, D. 1995. Structural genes for salicylate biosynthesis from chorismate in Pseudomonas aeruginosa. Mol. Gen. Genet. 249:217-228.

Shulaev, V., Leon, J., and Raskin, I. 1995. Is salicylic acid a translocated signal of systemic acquired resistance in tobacco? Plant Cell 7:16911701 .

Smith-Becker, J., Marois, E., Huguet, E. J., Midland, S. L., Sims, J. J., and Keen, N. T. 1998. Accumulation of salicylic acid and 4hydroxybenzoic acid in phloem fluids of cucumber during systemic acquired resistance is preceded by a transient increase in phenylalanine ammonia-lyase activity in petioles and stems. Plant Physiol. 116:231-238.

van Loon, L. C., Bakker, P. A. H. M., and Pieterse, C. M. J. 1998. Systemic resistance induced by rhizosphere bacteria. Annu. Rev. Phytopathol. 36:453-483

Vernooij, B., Friedrich, L., Morse, A., Reist, R., Kolditz-Jawhar, R., Ward, E., Uknes, S., Kessmann, H., and Ryals, J. 1994. Salicylic acid is not the translocated signal responsible for inducing systemic acquired resistance, but is required in signal transduction. Plant Cell 6:959-965. 